\title{
Investigative study of Withania somnifera family Solanaceae cultivated in Egypt to be used for pharmaceutical industry
}

\author{
Rahma mahrous, Hoda Fathy, Rasha Abu EL-Khair and Abdallah Omar
}

Department of Pharmacognosy, Faculty of Pharmacy, Alexandria, Egypt

\section{III}

Background: An investigative study of Egyptian W. somnifera revealed that the plant belongs to chemotype uiolll and having 20-hydroxywithanolides with $\alpha$-oriented side chain. Aim: Test whether Withania somnifera family Solanaceae cultivated in Egypt can be used for pharmaceutical industry. Materials and Methods: An accurate TLC method was developed and is introduced for the evaluation of this chemotype. Cell viability assay showed that the ethanolic extracts of leaves produce significant anticancer effect in liver and breast cancer cell lines. Results: Withanolides $E$ and $C$ had the highest effects against liver cancer. Moreover, withanolide $\mathrm{C}$ is pointed out as a lead anticancer agent targeting both breast and liver cancer cell lines. Ripe fruit extract showed the best inhibitory activity towards AChE as well as moderate antioxidant and nitric-oxide scavenging activities which explain the therapeutic potential of this extract in treatment of Alzheimer's. Withanolide S; a major constituent in the Egyptian plant that was also found in the leaves of the Italian and Israelian plant, could be regarded as an interesting drug candidate for treatment of Alzheimer's disease based on its strong (AChE) inhibitory activity. Conclusion: All together it can be concluded that unlike the Indian plant that is reputed for the medicinal use of its roots, both the fruits and leaves of Egyptian plant have better biological activities than the roots. Towards improving the extraction efficiency of withanolides, we suggest the use of soxhlet extraction as the optimum extraction method for industrial application using $70 \%$ ethanol for extraction of dried powdered leaves.

Keywords: Alzheimer's; Anticancer; Withanolides; Withania somnifera

Editor-in-Chief: Prof. M.L. Salem, PhD - Article DOI: 10.21608/jcbr.2021.58552.1124 